\title{
Optimal Operation of a CCHP Microgrid Using Interval Mixed-Integer Linear Programming
}

\author{
Zhao Luo, Wei Gu, Song Gao, Zhihe Wang, and Yiyuan Tang
}

\begin{abstract}
The development of combined cooling, heating, and power (CCHP) microgrids using distributed cogeneration equipment and renewable energy sources has drawn considerable research attention. In this study, we propose an interval mixed-integer linear programming (IMILP) dispatching model for supporting the economic dispatch of a CCHP microgrid under uncertainties. Our model is based on the interval programming method, where uncertainties can be incorporated and communicated into the economic dispatch problem using interval values. The proposed IMILP dispatching model is decomposed into two sets of deterministic sub-models, $f^{-}$and $f^{+}$, which are solved sequentially. We use a case study of a hotel in China to demonstrate how this model can be applied to support economic dispatch decisions for a CCHP microgrid. Simulation results indicated that load and PV power uncertainties mainly affect the dispatching results of the power exchange, gas boiler and electricity chiller, while the power generation unit is consistent under different uncertainty levels. The obtained dispatching results can provide more flexibility for decision makers by generating alternative decisions for CCHP microgrid dispatching under uncertainties.
\end{abstract}

Index Terms-CCHP, economic dispatch, interval programming, microgrid, uncertainty.

\section{INTRODUCTION}

The energy crisis and rising air pollution have led to greater worldwide focus on energy efficiency methods. Combined cooling, heating, and power (CCHP) systems are also referred to tri-generation systems. They have been widely used in small-medium scale power systems to solve energy-related problems, including increasing energy demands, higher energy costs, energy supply security, and environmental concerns [1]-[6]. A CCHP microgrid integrates power generation units (PGUs, e.g. Fuel cell or Micro turbine), auxiliary boilers, heat recovery systems, refrigeration equipment (electric chiller or absorption chiller), energy storage devices (heat storage tanks or storage batteries), and renewable energy sources (e.g. photovoltaic, wind, biomass) to implement the cascade utilization of energy. The main difference between CCHP microgrid and traditional CCHP systems is that the former can not only satisfy the cooling,

Manuscript received August 28, 2015; revised February 25, 2016. This work was supported by the National High Technology Research and Development Program of China (863 Program, grant no. 2011AA05A107), the National Science Foundation of China (grant no. 51277027), the Natural Science Foundation of Jiangsu Province of China (grant no. SBK201122387), the State Grid Corporation of China.

Zhao Luo, Wei Gu, Zhihe Wang, and Yiyuan Tang are with the School of Electrical Engineering, Southeast University, Nanjing, Jiangsu 210096, China (e-mail: waiting.1986@live.com).

Song Gao is with Jiangsu Electric Power Company, Nanjing, Jiangsu 210024, China. heating, and power demands of certain types of customers (such as office buildings, hotels, schools, commercial malls, and industrial loads), but also interacts with the main grid to provide reserve, peak-saving, demand response services, and provides improved capabilities for integrating renewable energy sources [7].

A CCHP microgrid system undoubtedly offers the end-users increased energy provision flexibility and has other merits. This type of system has a promising future, but there are some obstacles. A CCHP microgrid with a renewable energy source is a complex system with many uncertainties, a variety of structures, and highly coupled characteristics caused by the physical connections between components. Variations in weather conditions and the inexactness of predictive models combine to add randomness to both the load profile and renewable energy power output. It can also be difficult to characterize the performance of the components because of the nonlinearity of their efficiency curves, which cannot be expressed as deterministic values. Considering these problems, it is not easy to optimize the operation of a CCHP microgrid.

A review by Gu et al. [7] summarizes current research into the economic dispatch of CCHP microgrids [8]-[11]. Previous work has dealt with uncertainties in CCHP microgrid energy management using stochastic and fuzzy programs, or quantitative uncertainty analysis. The stochastic multi-objective model for cogeneration dispatch minimizes the expected production costs, the expected power generation deviation, and the expected heat generation deviation [12]-[16]. The chance constrained programming approach has been applied to solve uncertainty problems [17]-[19]. Moradi et al. [20] applied fuzzy programing to energy management system strategies for combined heat and power systems. Another approach for energy management optimization under uncertainty for CCHP microgrids is based on quantitative uncertainty analysis. Smith et al. presented an analysis of a CCHP system model under different operating strategies, with input data uncertainty [21]. Rezvan et al. presented a model to determine the capacity of distributed generators under uncertainty in load demand [22]. Generally, the above methods have advantages when dealing with uncertainties. However, there are difficulties when acquiring probability density functions (PDFs) for a number of the modeling parameters in stochastic methods, and the fuzzy programing and quantitative uncertainty analysis techniques need precise data parameters [23].

The complex models of previous studies limited their practical applicability to real-world cases. There are simplified models in a few studies, but most were designed to be run by dispatchers with the necessary background 
knowledge. Thus, the outputs of these dispatch efforts were highly subjective and dependent on ability, knowledge, and dispatcher understandings. This not only prevents extensive application, but also affects their accuracy and reliability and possibly makes them unsuitable for use by decision makers. Therefore, it is necessary to develop effective solutions for economic dispatch in the CCHP microgrid under various uncertainties and dynamic characteristics, which can be easily used by decision makers under multiple time scales [24]-[26].

Uncertain decision variables cannot be reflected with deterministic values. In fact, the uncertainties of many practical problems may be expressed as ambiguous intervals, because dispatchers find it easier to define fluctuation intervals than deterministic values and PDFs. Interval programing is particularly convenient for describing volatile power output, instead of using a fixed prediction or a presumed probability distribution [27]. Another equally important problem in the CCHP microgrid model is how to use interval programing.

Therefore, the objective of this study was to develop an interval mixed-integer linear programming (IMILP) model [28] for the economic dispatch of a CCHP microgrid. We analyzed and interpreted the optimal interval solutions for both binary and continuous variables to provide useful decision and application information. To the authors' best knowledge, the day-ahead economic dispatch of a CCHP microgrid using an interval programing approach has not been previously investigated in the literature.

This paper is organized as follows. Section II proposes an IMILP model to implement the economic dispatch of a CCHP microgrid with uncertainty. Section III presents a decomposition strategy for the IMILP model. In Section IV, we have tested the proposed approach in a real system to demonstrate its capability in providing decision support for a CCHP microgrid under various uncertainties. Finally, we conclude this paper in Section V.

\section{OPtimal DisPatching MODEL FOR A CCHP MICROGRID}

A schematic of the energy flows for the CCHP microgrid is given in Fig. 1. In this paper, we consider a CCHP microgrid that includes a photovoltaic cell (PV), a battery, a PGU, a gas boiler, an absorption chiller, an electric chiller, and heat exchangers, along with cooling, heating and power loads.

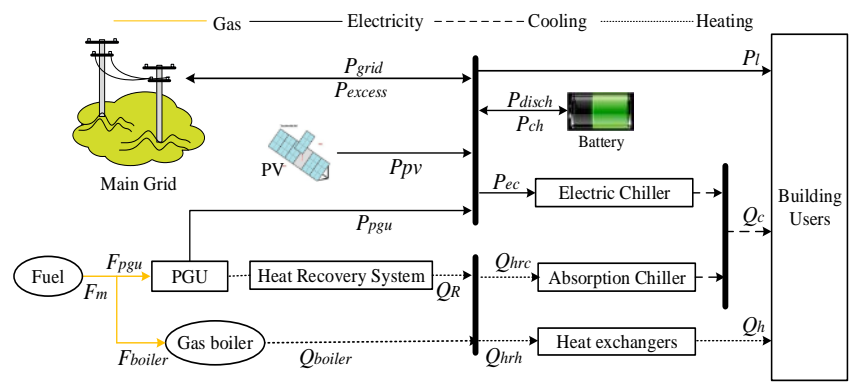

Fig. 1. Schematic of a CCHP microgrid.

The power between the CCHP microgrid and the main grid is bidirectional. Renewable energy resources and a PGU are used to generate electricity to supply the end user, and excess electricity can be sold back to the main grid or used to charge batteries. So the CCHP microgrid can purchase power from the main grid or sell power to the main grid according to the demands. The heat output from the PGU is recovered by the heat recovery system to produce cooling using an absorption chiller or heating using heat exchangers. When there is not enough recovered heat, the auxiliary boiler of the CCHP microgrid is used to provide extra heat. As the legends in Fig. 1 show, PV power and the cooling, heating and power loads are stochastic variables. The PGU power, battery, gas boiler, and proportion of the absorption cooling in the cooling loads are control variables. The absorption chiller power and the power between the electric chiller and the main grid are controlled variables.

\section{A. Modeling of the Components}

1) Power Generation Unit (PGU): The rated power of the prime mover is given, so the fuel consumption and waste heat supplied by the PGU can be determined by (1) and (2), respectively:

$$
\begin{gathered}
F_{p g u, t}^{ \pm}=P_{p g u, t}^{ \pm} / \eta_{p g u}^{ \pm} \\
Q_{R, t}^{ \pm}=F_{p g u, t}^{ \pm}\left(1-\eta_{p g u}^{ \pm}\right)=P_{p g u, t}^{ \pm}\left(1-\eta_{p g u}^{ \pm}\right) / \eta_{p g u}^{ \pm}
\end{gathered}
$$

2) Heat Recovery System: The recovered thermal energy from the prime mover can be estimated as:

$$
Q_{r e c, t}^{ \pm}=Q_{R, t}^{ \pm} \eta_{r e c}^{ \pm}=P_{p g u, t}^{ \pm}\left(1-\eta_{p g u}^{ \pm}\right) \eta_{r e c}^{ \pm} / \eta_{p g u}^{ \pm}
$$

3) Energy Storage Unit (battery): A CCHP microgrid with a battery can effectively shift the peak load and improve the operational efficiency of the cogeneration units. When the amount of electricity generated by the cogeneration units is greater than the electricity demands, the excess will charge the battery when the electricity price is at a relatively low price. It can also provide part of the electricity when the CCHP microgrid is not meeting the demand. This improves the economics of the CCHP microgrid.

$$
W_{E S, t}=W_{E S, t-1}(1-\mu)+\left(\eta_{c h} P_{c h, t}^{ \pm}-P_{d i s c h, t}^{ \pm} / \eta_{d i s c h}\right) \Delta t
$$

4) Gas boiler: The gas boiler generates heat. Its input power is

$$
F_{\text {boiler }, t}^{ \pm}=Q_{\text {boiler }, t}^{ \pm} / \eta_{\text {boliler }}^{ \pm}
$$

5) Chiller: The cooling capacity is mainly provided by the absorption and electric chillers. The absorption chiller is driven by the thermal power generated by the PGU and gas boiler. Its output power is

$$
Q_{a c, t}^{ \pm}=Q_{h r c, t}^{ \pm} \cdot C O P_{a c}^{ \pm}
$$

The output power of the electric chiller is

$$
Q_{e c, t}^{ \pm}=P_{e c, t}^{ \pm} \cdot C O P_{e c}^{ \pm}
$$


6) Heat exchangers: The output power of the heat exchangers is

$$
Q_{h, t}^{ \pm}=Q_{h r h, t}^{ \pm} \cdot \eta_{h x}^{ \pm}
$$

\section{B. Optimization Objective}

The objective of the IMILP is to minimize the total running costs. These include the cost of electricity purchased from the main grid and fuel costs for the PGU and gas boiler. The cost is offset by the profits from selling electricity to the main grid. The decision variables can be classified into two types: binary and continuous. The binary variables can be used to avoid negative values produced by the exchange power with the microgrid or battery. The continuous variables are used to reflect the energy-related activities of the CCHP microgrid components. In our study, interval values are used to describe the uncertainties of variables. Accordingly, the objective function can be formulated as:

$$
\operatorname{Minf}^{ \pm}=\sum_{t=1}^{T}\left[C_{\text {purchase }, t} \cdot P_{\text {grid }, t}^{ \pm}-C_{\text {sell }, t} \cdot P_{\text {excess }, t}^{ \pm}+C_{\text {gas }} \cdot F_{m, t}^{ \pm}\right] \Delta t
$$

Here, $F_{m, t}^{ \pm}=F_{p g u, t}^{ \pm}+F_{b o i l e, t}^{ \pm}=P_{p g u, t}^{ \pm} / \eta_{p g u}^{ \pm}+Q_{b o i l e, t}^{ \pm} / \eta_{b o i l e r}^{ \pm}, \Delta t$ is the period, and $T$ is the total number of periods. $C_{\text {purchase, }}$ is the tariff for purchasing power from the main grid $(\$ / \mathrm{kWh}), C_{\text {sell, }}$ is the tariff for selling power to the main grid $(\$ / \mathrm{kWh})$, and $C_{\text {gas }}$ is the tariff for natural gas $(\$ / \mathrm{kWh})$.

\section{System Constraints}

Constraints of the model include the cooling, heating, and power balances and the operational constraints of each component.

1) Energy balance constraints for cooling, heating and power

1) Power balance

$$
P_{g r i d, t}^{ \pm}+P_{p g u, t}^{ \pm}+P_{d i s c h, t}^{ \pm}-P_{e x c e s s, t}^{ \pm}-P_{c h, t}^{ \pm}-P_{e c, t}^{ \pm}=P_{l, t}^{ \pm}-P_{p v, t}^{ \pm}
$$

2) Thermal balance

$$
Q_{r e c, t}^{ \pm}=Q_{b o i l e r, t}^{ \pm}-Q_{h r c, t}^{ \pm}-Q_{h r h, t}^{ \pm} \geq 0
$$

3) Cold balance

$$
Q_{a c, t}^{ \pm}+Q_{e c, t}^{ \pm} \geq Q_{c, t}^{ \pm}
$$

\section{2) Component capacity constraints}

1) PGU constraints

$$
P_{p g u}^{\min } \leq P_{p g u, t}^{ \pm} \leq P_{p g u}^{\max }
$$

where $P_{p g u}^{\min }$ and $P_{p g u}^{\max }$ are the minimum and maximum power efficiencies of the PGU $(\mathrm{kW})$.

2) Gas boiler constraints

$$
Q_{b o i l e r}^{\min } \leq Q_{b o i l e r, t}^{ \pm} \leq Q_{b o i l e r}^{\max }
$$

where $Q_{b o i l e r}^{\min }$ and $Q_{b o i l e r}^{\max }$ are the minimum and maximum power efficiencies of the Gas boiler $(\mathrm{kW})$.

3) Heat recovery system constraints

$$
Q_{R}^{\min } \leq Q_{R, t}^{ \pm} \leq Q_{R}^{\max }
$$

where $Q_{R}^{\min }$ and $Q_{R}^{\max }$ are the minimum and maximum power efficiencies of the gas boiler $(\mathrm{kW})$.

4) Electric chiller constraints

$$
P_{e c}^{\min } \leq P_{e c, t}^{ \pm} \leq P_{e c}^{\max }
$$

where $P_{e c}^{\min }$ and $P_{e c}^{\max }$ are the minimum and maximum power efficiencies of the electric chiller $(\mathrm{kW})$.

5) Absorption chiller constraints

$$
Q_{a c}^{\min } \leq Q_{a c, t}^{ \pm} \leq Q_{a c}^{\max }
$$

where $Q_{a c}^{\min }$ and $Q_{a c}^{\max }$ are the minimum and maximum power efficiencies of the absorption chiller $(\mathrm{kW})$.

6) Heat exchanger constraints

$$
Q_{h r h}^{\min } \leq Q_{h r h, t}^{ \pm} \leq Q_{h r h}^{\max }
$$

where $Q_{h r h}^{\min }$ and $Q_{h r h}^{\max }$ are the minimum and maximum power efficiencies of the gas boiler $(\mathrm{kW})$.

3) Battery constraints

$$
\left\{\begin{array}{c}
0 \leq P_{c h, t}^{ \pm} \leq U_{c h, t}^{ \pm} P_{c h}^{\max }=U_{c h, t}^{ \pm} \operatorname{Cap}_{E S} \gamma_{E S, c h} \\
0 \leq P_{d i s c h, t}^{ \pm} \leq U_{d i s c h, t}^{ \pm} P_{d i s c h}^{\max }=U_{d i s c h, t}^{ \pm} \operatorname{Cap}_{E S} \gamma_{E S, \text { disch }} \\
U_{c h, t}^{ \pm}+U_{d i s c h, t}^{ \pm} \leq 1 \\
W_{E S}^{\min } \leq W_{E S, t} \leq W_{E S}^{\max }
\end{array}\right.
$$

where $W_{E S}^{\min }$ and $W_{E S}^{\max }$ are the minimum and maximum power of the battery $(\mathrm{kW})$. The energy in the battery at the initial interval and terminal interval is fixed to be the same. $U_{\text {disch, }}^{ \pm}$is the $0-1$ state variable of the battery discharge at time $t$ ( 0 for stopped, 1 for discharging) and $U_{c h, t}^{ \pm}$is the 0 - 1 state variable of the battery charge at time $t$ ( 0 for stopped, 1 for charging).

4) Constraints of the power exchange between the CCHP and main grid

$$
\left\{\begin{array}{c}
U_{P, t}^{ \pm} P_{\text {grid }}^{\min } \leq P_{\text {grid }, t}^{ \pm} \leq U_{P, t}^{ \pm} P_{\text {grid }}^{\max } \\
U_{S, t}^{ \pm} P_{\text {excess }}^{\min } \leq P_{\text {excess }, t}^{ \pm} \leq U_{S, t}^{ \pm} P_{\text {excess }}^{\max } \\
U_{P, t}^{ \pm}+U_{S, t}^{ \pm} \leq 1
\end{array}\right.
$$

where $P_{\text {grid }}^{\max }$ and $P_{\text {grid }}^{\min }$ are the maximum and minimum exchange power between the CCHP and main grid, that is the maximum power purchased from, and sold to, the main grid $(\mathrm{kW}) . U_{P, t}^{ \pm}$ represents whether the CCHP microgrid purchases power from the main grid in period $t$ ( 0 for not purchasing, 1 for purchasing), and $U_{S, t}^{ \pm}$represents whether the CCHP microgrid sells electricity from the main grid in period $t$ ( 0 for not selling, 1 for selling). 


\section{IMILP SOLUTION METHOD}

The above economic dispatch model of a CCHP microgrid is an IMILP model. According to Huang [29], Li et al. [30], it can be solved by decomposing it into two sets of deterministic sub-models. Using interval computation, in the minimum operating condition $\left(O C^{-}\right)$, the $\mathrm{PV}$ power is $P_{p v, t}^{+}$, and the electricity, heating, and cooling demands are $P_{l, t}^{-}, Q_{h, t}^{-}, Q_{c, t}^{-}$In the maximum operating condition $\left(O C^{+}\right)$, the $\mathrm{PV}$ power is $P_{p v, t}^{-}$ the electricity, heating, and cooling demands are $P_{l, t}^{+}, Q_{h, t}^{+}, Q_{c, t}^{+}$. Using the IMILP solution, we have the sub-models, $f^{-}$and $f^{+}$. Model $f^{-}$represents the minimum running cost of $\mathrm{OC}^{-}$, and $\mathrm{f}^{+}$represents the minimum running cost of $\mathrm{OC}^{+}$. The resulting solution provides intervals for the objective function and alternative decisions. Details are described as follows.

$f^{-}$sub-model:



$$
\begin{aligned}
& \text { s.t. } P_{g r i d, t}^{-}+P_{p g u, t}^{-}+P_{d i s c h, t}^{-}-P_{e x c e s s, t}^{+}-P_{c h, t}^{-}-P_{e c, t}^{-}=P_{l, t}^{-}-P_{p v, t}^{+} \text {, } \\
& Q_{\text {rec }, t}^{-}+Q_{\text {boiler }, t}^{-}-Q_{h r c, t}^{-}-Q_{h r h, t}^{-} \geq 0, \\
& Q_{h r h}^{-} \geq Q_{h, t}^{-} / \eta_{h r h}^{+} ; Q_{a c, t}^{-}+Q_{e c, t}^{-} \geq Q_{c, t}^{-}, \\
& U_{P, t}^{-} P_{e x}^{\min } \leq P_{\text {grid }, t}^{-} \leq U_{P, t}^{-} P_{e x}^{\max } ; U_{S, t}^{+} P_{e x}^{\min } \leq P_{\text {excess }, t}^{+} \leq U_{S, t}^{+} P_{e x}^{\max } \\
& U_{P, t}^{-}+U_{S, t}^{+} \leq 1 ; P_{p g u}^{\min } \leq P_{p g u, t}^{-} \leq P_{p g u}^{\max }, \\
& Q_{\text {boiler }}^{\min } \leq Q_{\text {boiler }, t}^{-} \leq Q_{\text {boiler }}^{\max } ; Q_{R}^{\min } \leq Q_{R, t}^{-} \leq Q_{R}^{\max }, \\
& Q_{a c}^{\min } \leq Q_{a c, t}^{-} \leq Q_{a c}^{\max } ; Q_{h r h}^{\min } \leq Q_{h r h, t}^{-} \leq Q_{h r h}^{\max }, \\
& 0 \leq P_{c h, t}^{-} \leq U_{c h, t}^{-} P_{c h}^{\max } ; 0 \leq P_{\text {disch }, t}^{-} \leq U_{\text {disch }, t}^{-} P_{\text {disch }}^{\max }, \\
& U_{c h, t}^{-}+U_{\text {disch }, t}^{+} \leq 1 ; W_{E S}^{\min } \leq W_{E S, t} \leq W_{E S}^{\max }, \\
& W_{E S, t}=W_{E S, t-1}(1-\mu)+\left(\eta_{c h} P_{c h, t}^{-}-P_{d i s c h, t}^{-} / \eta_{d i s c h}\right), \\
& 0 \leq U_{P, t}^{-} \leq 1 \text { and } U_{P, t}^{-} \text {is an integer, } \\
& 0 \leq U_{S, t}^{+} \leq 1 \text { and } U_{S, t}^{+} \text {is an integer, } \\
& 0 \leq U_{c h, t}^{-} \leq 1 \text { and } U_{c h, t}^{-} \text {is an integer, }
\end{aligned}
$$

$$
0 \leq U_{\text {disch,t }}^{-} \leq 1 \text { and } U_{\text {disch,t }}^{-} \text {is an integer. }
$$

The $f_{\text {opt }}^{-}$and decision variables can be obtained from the solution corresponding to $f^{-}$. The decision variable's solutions can provide constraints for $f^{+}$, which can be formulated as follows:

$$
f^{+} \text {sub-model: }
$$

$$
\begin{aligned}
& \mathrm{Minf}^{+}=\sum_{t=1}^{T}\left[C_{\text {grid }, t} \cdot P_{\text {grid }, t}^{+}-C_{\text {grid }, t} P_{\text {excess }, t}^{-}+C_{\text {gas }} \cdot\left(\frac{P_{p g u, t}^{+}}{\eta_{p g u}^{-}}+\frac{Q_{\text {boiler }, t}^{+}}{\eta_{\text {boiler }}^{-}}\right)\right] \Delta t \\
& \text { s.t. } P_{g r i d, t}^{+}+P_{p g u, t}^{+}+P_{d i s c h, t}^{+}-P_{e x c e s s, t}^{-}-P_{c h, t}^{+}-P_{e c, t}^{+}=P_{l, t}^{+}-P_{p v, t}^{-} \text {, } \\
& Q_{\text {rec }, t}^{+}+Q_{\text {boiler }, t}^{+}-Q_{h r c, t}^{+}-Q_{h r h, t}^{+} \geq 0, \\
& Q_{h r h}^{+} \geq Q_{h, t}^{+} / \eta_{h r h}^{-} ; Q_{a c, t}^{+}+Q_{e c, t}^{+} \geq Q_{c, t}^{+}, \\
& U_{P, t}^{+} P_{e x}^{\min } \leq P_{\text {grid }, t}^{+} \leq U_{P, t}^{+} P_{e x}^{\max } ; U_{S, t}^{-} P_{e x}^{\min } \leq P_{\text {excess }, t}^{-} \leq U_{S, t}^{-} P_{e x}^{\max }, \\
& U_{P, t}^{+}+U_{S, t}^{-} \leq 1 ; P_{p g u}^{\min } \leq P_{p g u, t}^{+} \leq P_{p g u}^{\max }, \\
& Q_{\text {boiler }}^{\min } \leq Q_{\text {boiler }, t}^{+} \leq Q_{\text {boiler }}^{\max } ; Q_{R}^{\min } \leq Q_{R, t}^{+} \leq Q_{R}^{\max }, \\
& Q_{a c}^{\min } \leq Q_{a c, t}^{+} \leq Q_{a c}^{\max } ; Q_{h r h}^{\min } \leq Q_{h r h, t}^{+} \leq Q_{h r h}^{\max }, \\
& 0 \leq P_{c h, t}^{+} \leq U_{c h, t}^{+} P_{c h}^{\max } ; 0 \leq P_{d i s c h, t}^{+} \leq U_{d i s c h, t}^{+} P_{d i s c h}^{\max },
\end{aligned}
$$

$$
\begin{aligned}
& U_{c h, t}^{+}+U_{d i s c h, t}^{+} \leq 1 ; W_{E S}^{\min } \leq W_{E S, t} \leq W_{E S}^{\max }, \\
& W_{E S, t}=W_{E S, t-1}(1-\mu)+\left(\eta_{c h} P_{c h, t}^{+}-P_{d i s c h, t}^{+} / \eta_{d i s c h}\right), \\
& P_{g r i d, t}^{+} \geq P_{g r i d, t}^{-} ; P_{p g u, t}^{+} \geq P_{p g u, t}^{-} ; P_{\text {excess }, t}^{-} \leq P_{\text {excess }, t}^{+}, \\
& P_{d i s c h, t}^{+} \geq P_{d i s c h, t}^{-} ; P_{c h, t}^{+} \geq P_{c h, t}^{-} ; P_{e c, t}^{+} \geq P_{e c, t}^{-}, \\
& Q_{b o i l e r, t}^{+} \geq Q_{b o i l e r, t}^{-} ; Q_{a c, t}^{+} \geq Q_{a c, t}^{-} ; Q_{r e c, t}^{+} \geq Q_{r e c, t}^{-}, \\
& P_{d i s c h, t}^{+} \geq P_{d i s c h, t}^{-} ; P_{c h, t}^{+} \geq P_{c h, t}^{-}, \\
& U_{P, t}^{+} \geq U_{P, t}^{-} ; U_{S, t}^{-} \geq U_{S, t}^{+}, \\
& U_{P, t}^{+} \geq U_{P, t}^{-} ; U_{S, t}^{-} \geq U_{S, t}^{+} ; U_{c h, t}^{+} \geq U_{c h, t}^{-} ; U_{d i s c h, t}^{-} \geq U_{d i s c h, t}^{+}, \\
& 0 \leq U_{P, t}^{+} \leq 1 \text { and } U_{P, t}^{+} \text {is an integer, } \\
& 0 \leq U_{S, t}^{-} \leq 1 \text { and } U_{S, t}^{-} \text {is an integer, } \\
& 0 \leq U_{c h, t}^{+} \leq 1 \text { and } U_{c h, t}^{+} \text {is an integer, } \\
& \quad 0 \leq U_{d i s c h, t}^{-} \leq 1 \text { and } U_{d i s c h, t}^{-} \text {is an integer. }
\end{aligned}
$$

Each of the sub-models $f^{-}$and $f^{+}$is an ordinary mixed-integer linear programming (MILP) problem with a single objective function. The solutions to these MILP problems can be obtained using existing commercial software. Generally, the solution of the IMILP model can be calculated as follows.

Step 1: Formulate the IMILP model.

Step 2: Transform the IMILP model into two sub-models. Step 3: Formulate the $f^{-}$sub-model.

Step 4: Solve the $f^{-}$sub-model and obtain $f_{\text {opt }}^{-}, P_{\text {grid, }, \text { opt }}^{-}$,

$$
\begin{aligned}
& P_{\text {pgut, }, \text { opt }}^{-}, P_{\text {excess }, \text { opt }}^{+}, P_{\text {disch }, t, o p t}^{-}, P_{c h, t, o p t}^{-}, P_{\text {ec, }, \text { opt }}^{-}, Q_{\text {boiler }, \text { opt }}^{-}, \\
& Q_{a c, t, \text { opt }}^{-}, Q_{\text {rec, }, \text { opt }}^{-}
\end{aligned}
$$

Step 5: Formulate the $f^{+}$sub-model based on the solution of the $f^{-}$sub-model.

Step 6: Solve the $f^{+}$sub-model and obtain $f_{\text {opt }}^{+}, P_{\text {grid,t,opt }}^{+}$,

$$
P_{p s u, t, o p t}^{+}, P_{\text {excess }, \text { opt }}^{-}, P_{\text {disch }, \text { opt }}^{+}, P_{c h, t, o p t}^{+}, P_{\text {ec, }, \text { opt }}^{+}, Q_{b o i l e r, t, o p t}^{+},
$$$$
Q_{a c, t, o p t}^{+}, Q_{\text {rec, }, \text { opt }}^{+} \text {. }
$$

Step 7: Obtain the solutions of the IMILP model using

$$
\begin{aligned}
& f_{\text {opt }}^{ \pm}=\left[f_{\text {opt }}^{-}, f_{\text {opt }}^{+}\right], \\
& P_{\text {grid, }, \text { opt }}^{ \pm}=\left[P_{\text {grid, }, \text { opt }}^{-}, P_{\text {grid, }, \text { opt }}^{ \pm}\right], \\
& P_{p g u, t, o p t}^{ \pm}=\left[P_{p g u t, \text { opt }}^{-}, P_{p g u, t, o p t}^{+}\right], \\
& P_{\text {excess }, \text { topt }}^{ \pm}=\left[P_{\text {excess }, t, o p t}^{-}, P_{\text {excess }, \text { opt }}^{+}\right] \text {, } \\
& P_{\text {disch, }, \text { opt }}^{ \pm}=\left[P_{\text {disch,t,opt }}^{-}, P_{\text {disch, }, \text { opt }}^{+}\right] \text {, } \\
& P_{c h, t, o p t}^{ \pm}=\left[P_{c h, t, o p t}^{-}, P_{c h, t, o p t}^{+}\right], \\
& P_{e c, t o p t}^{ \pm}=\left[P_{e c, t, o p t}^{-}, P_{e c, t, o p t}^{+}\right], \\
& Q_{\text {boiler,t,opt }}^{ \pm}=\left[Q_{\text {boiler,t,opt }}^{-}, Q_{\text {boiler, }, \text { opt }}^{+}\right], \\
& Q_{a c, t, o p t}^{ \pm}=\left[Q_{a c, t, o p t}^{-}, Q_{a c, t, o p t}^{+}\right], \\
& Q_{\text {rec, }, \text { opt }}^{ \pm}=\left[Q_{\text {rec }, t, o p t}^{-}, Q_{\text {rec, }, \text { opt }}^{+}\right] \text {. }
\end{aligned}
$$

\section{CASE STUdy}

We tested the proposed IMILP model and solution method on a typical CCHP microgrid to demonstrate its applicability. This CCHP microgrid belongs to a hotel in Beijing, China. The heating demands are provided for by natural gas, and the cooling demands are mainly provided for by heat and electricity. The dispatcher's major concern is to investigate the operating economy of the microgrid during the entire schedule horizon under uncertainties. We estimated the energy consumption of some representative days using the 
Energy Plus-simulation software [31]. Fig. 2 shows the hourly PV power and the cooling, heating, and power loads for representative days in winter and summer.

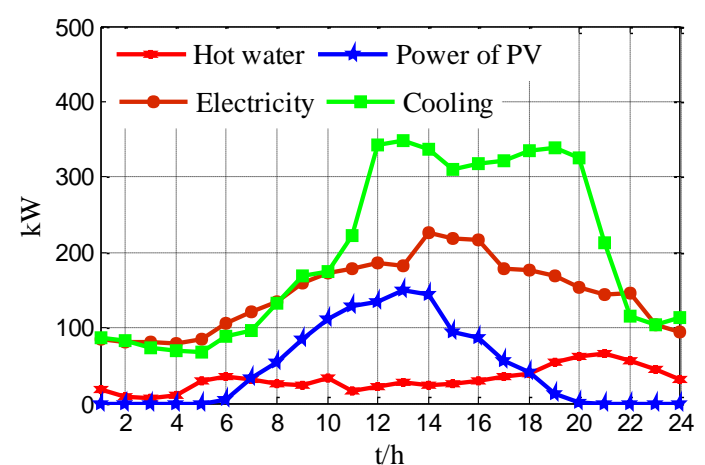

(a)

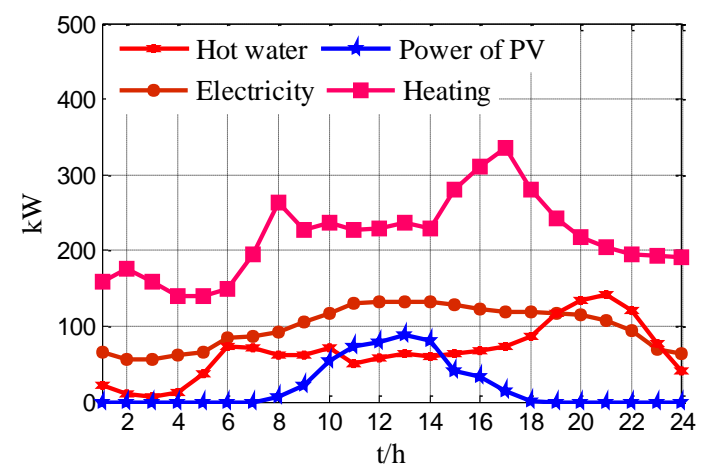

(b)

Fig. 2. Hourly PV power, and cooling, heating, and power loads of the hotel in representative days of summer (a) and winter (b).

\begin{tabular}{ccc}
\multicolumn{3}{c}{ TABLE I: ELECTRICITY PRICES } \\
\hline \hline Time & $\begin{array}{c}\text { Purchase } \\
\text { price } \$ / \mathrm{kWh})\end{array}$ & $\begin{array}{c}\text { Sell } \\
\text { price } \$ / \mathrm{kWh})\end{array}$ \\
\hline 0:00-7:59 & 0.11 & 0.085 \\
$8: 00-13: 59$ & 0.15 & 0.12 \\
14:00-17:59 & 0.11 & 0.085 \\
$18: 00-21: 59$ & 0.15 & 0.12 \\
$22: 00-23: 59$ & 0.11 & 0.085 \\
\hline \hline
\end{tabular}

TABLE II: CAPACITIES AND COEFFICIENTS OF THE FACILITIES IN CCHP MICRO-GRID

\begin{tabular}{ccc}
\hline \multicolumn{3}{c}{ MICRO-GRID } \\
\hline Facility & $\begin{array}{c}\text { Capacity } \\
(\mathrm{kW})\end{array}$ & Efficiency \\
\hline Prime mover (PGU) & 120 & $\eta_{p g u=}[0.28,0.3]$ \\
Gas boiler & 400 & $\eta_{\text {outs }}=[0.75,0.8]$ \\
Electric chiller & 100 & $C O P_{e c}=[3.8,4]$ \\
Absorption chiller & 500 & $C O P_{a c}=[0.68,0.7]$ \\
Heat exchangers & 600 & $\eta_{h x}=[0.87,0.9]$ \\
Heat Recovery System & 300 & $\eta_{r e c}=[0.72,0.75]$ \\
Battery & 200 & $\mu=0.04$ \\
Exchange Power & 300 & $\eta_{c h}=\eta_{\text {disch }}=0.95$ \\
PV & 189 & $/$ \\
\hline \hline
\end{tabular}

We carried out simulations of the proposed model and solution using MATLAB 7.1. Table I shows purchasing and selling prices at different times of the day. We chose natural gas as the fuel, which is widely used in CCHP microgrids. The price of natural gas is $0.4399 \$ / \mathrm{m}^{3}$, which is $0.0463 \$ / \mathrm{kWh}$ when converted into calorific units. The initial facilities' capacities for optimization are chosen according to the design of this building and the efficiency interval parameters of the equipment, which are listed in Table II.

In the CCHP microgrid, PV power, cooling, heating, and power load are stochastic variables. In practical applications, their uncertainties are not fixed. In addition, different seasonal loads can significantly affect the operating condition of the cogeneration equipment. These factors could all influence the dispatching results of the CCHP microgrid. In this paper, we have investigated three scenarios to address these issues. Scenarios 1 (summer) and 2 (winter) have $10 \%$ uncertainties on the load and PV. Scenario 3 has different uncertainties on the load and PV in summer and winter. We used the proposed method to solve the three scenarios.

\section{A. Interval Optimization With $10 \%$ Uncertainty in Summer}

Fig. 3(a)-(c) represent the optimization results for the minimum operating condition $O C_{10 \%}^{-}$in summer. The running cost is $\$ 355.9$. The PGU operates over the entire schedule horizon. Fig. 2 (a) shows that cooling load rapidly increases in Periods 9 to 14 , when the purchase price of electricity is high. Thus, the PGU works at its rated power so that the CCHP microgrid is efficient. The waste heat of the PGU first satisfies the hot water demands, and then the extra heat is supplied to the absorption chiller. Any cooling insufficiencies are provided by the electric chiller. The battery charges during Periods 15 to 18 at a low purchasing price, and discharges in Periods 19 to 20 at a high selling price. In summer, the power generated by the PV is larger than during other seasons, so the system gives the excess power back to the main grid to increase profits.

Fig. 3(d)-(f) represent the schedule of the maximum operating condition $O_{10 \%}^{+}$in summer. The running cost is \$549.0. In all time periods, the scheduling curves of the PGU and battery are extremely similar for both $O C_{10 \%}^{-}$and $O C_{10 \%}^{+}$. PGU provides a little more heating in Periods 5 to 8,15 to 18 , and 23 to 24 than in $O C_{10 \%}^{-}$, and suppresses the partial heating fluctuations. Other heating fluctuations are restrained by the gas boiler, which provides extra heat in Periods 9 to 14 and 19 to 22. To control electricity load fluctuations, the CCHP microgrid sells less of the extra power to the main grid in Periods 9 to 14 , and at other scheduling intervals the microgrid purchases more power from the main grid. When the variation magnitudes of the loads and PV do not exceed $10 \%$ of the predictive value, the scheduling results of the CCHP microgrid should be between $O C_{10 \%}^{-}$and $O C_{10 \%}^{+}$.

\section{B. Interval Optimization With $10 \%$ Uncertainty in Winter}

Fig. 4(a) and Fig. 4(b) show the optimization results for the minimum operating condition $O C_{10 \%}^{-}$in winter. The running cost is $\$ 394.2$. The battery charges in Periods 5 to 8 and 15 to 18 at a low purchasing price, and discharges in Periods 9 to 10 and 19 using the high selling price to maximize profits. As the heat needs are higher in winter, they can only be supplied by the PGU and gas boiler. The PGU remains working over 24 intervals to meet the thermal demands, and any extra heat requirements are satisfied by the gas boiler. Meanwhile, the extra power generated by the PGU is sold to the main grid or used to charge the battery, depending on the electricity price and state of battery. 

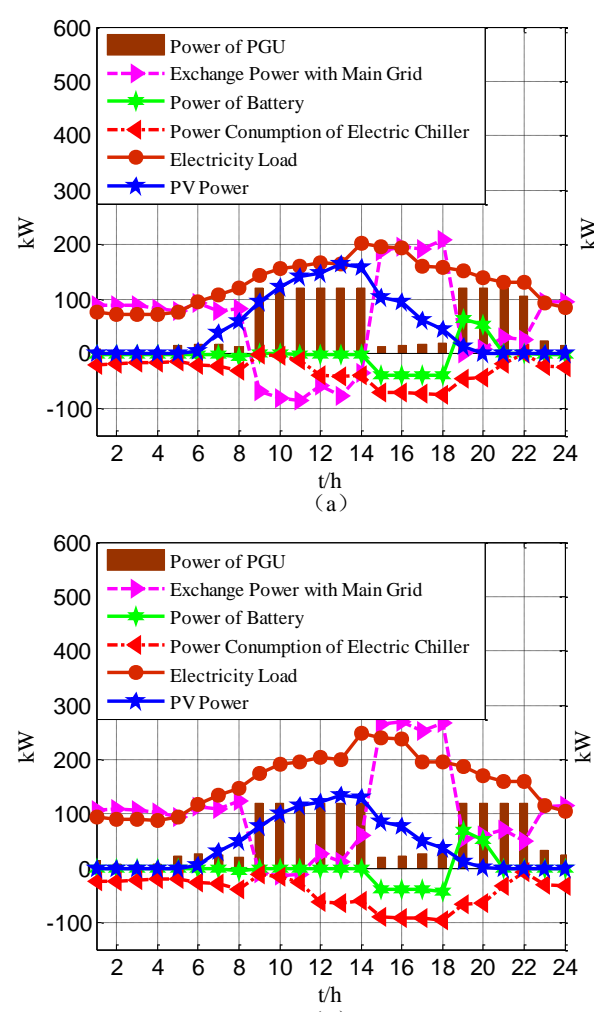

(d)

Fig. 3. Summer seasons curves for $O C_{10 \%}^{-}$: (a) electricity pow
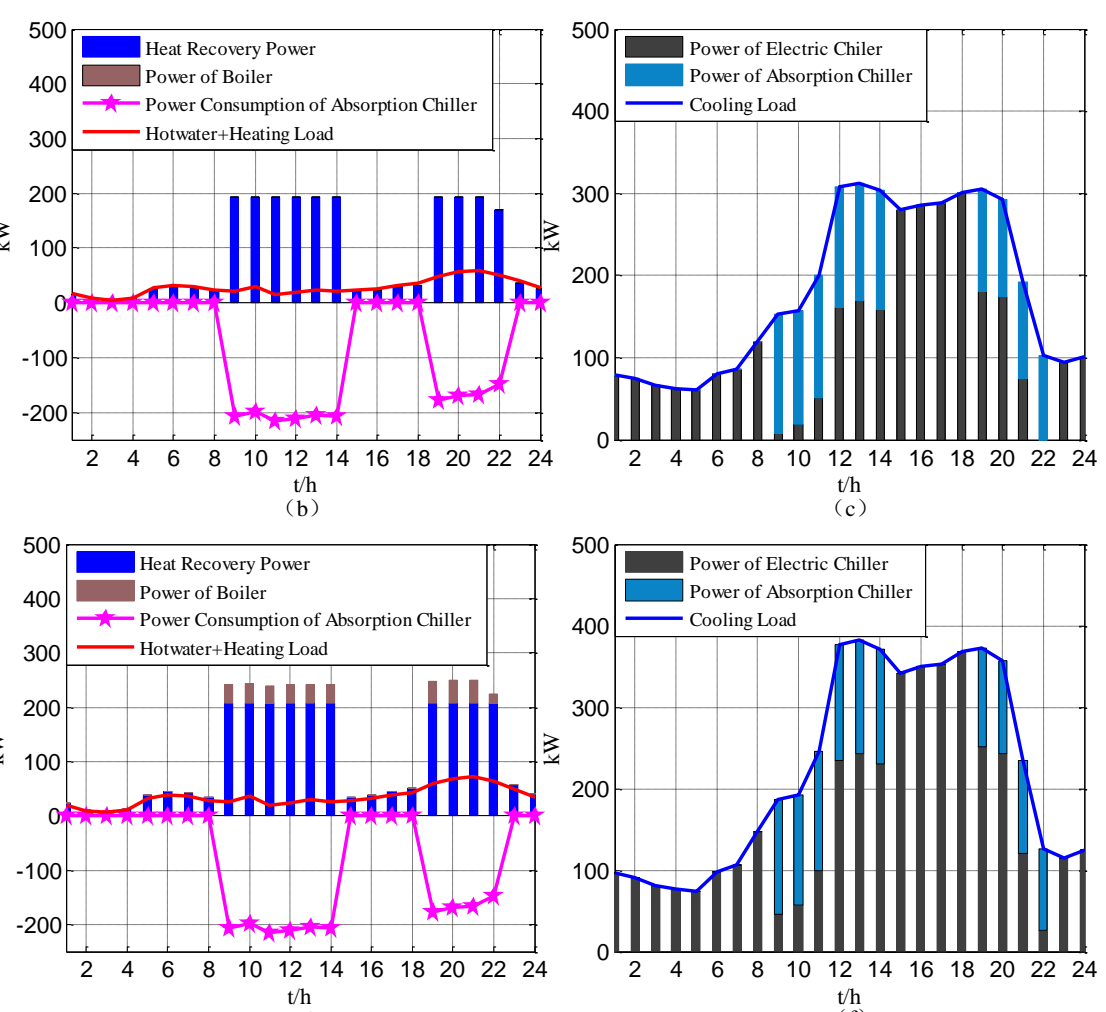

(e)

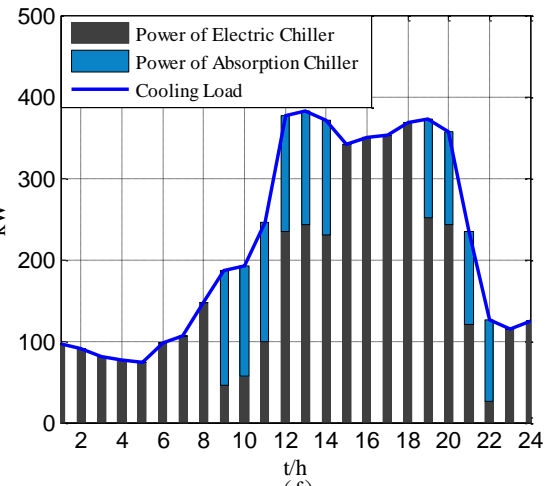

t/h

(e) heating power; (f) cooling power.

Fig. 4(c) and Fig. 4(d) show the schedule for the maximum operating condition $O C_{10 \%}^{+}$in winter. The running cost is $\$ 644.3$. In all time periods, the scheduling results of the PGU and battery are subtly different for $O C_{10 \%}^{-}$and $O C_{10 \%}^{+}$. In $O C_{10 \%}^{+}$, the PGU starts to provide its rated power in Period 5, but it starts in Period 6 in $O C_{10 \%}^{-}$. Most of the system's heating fluctuations are suppressed by the gas boiler, which starts in Periods 2 to 6 and provides more heat at other scheduling periods in $O C_{10 \%}^{+}$. To cope with electricity load fluctuations, the CCHP microgrid sells less of its extra power to the main grid during all scheduling periods, and purchases more power from the main grid over Periods 6 to 8 and 15 to 18 .

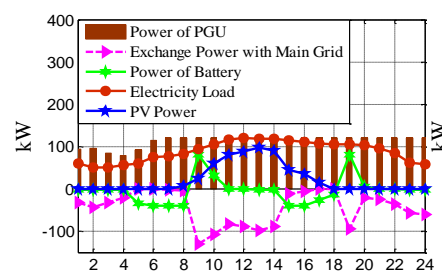

(a)

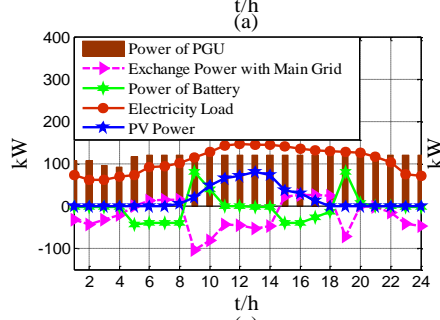

$\mathrm{t} / \mathrm{h}$

Fig. 4. Winter season curves for $O C_{10 \%}^{-}$: (a) Electricity power; (b) Heating power. Winter season curves for $O C_{10 \%}^{+}$: (c) Electricity power; (d) Heating power.

A comprehensive analysis of the two typical days shows that there are different dispatching results for the CCHP microgrid. In summer, we should use the electric chiller to meet the partial cooling demands and consume the excess power instead of selling it the main grid. However, in winter, the PGU generates the maximum possible amount of heat to satisfy the thermal demands. Meanwhile, the extra power is sold to the main grid or used to charge the battery, because the heat-electricity load ratio is larger than the thermoelectric ratio of the PGU.

\section{Impact of Different Uncertainty Levels}

The analysis above indicates that uncertainty has a great effect on the dispatching results of the CCHP microgrid. To more clearly illustrate the feasibility and effectiveness of the proposed IMILP model, we have used predictive values for representative summer and winter days to calculate and compare the running costs under 5\%, 10\%, and $15 \%$ uncertainties. The corresponding running costs are listed in Table III.

TABLE III: RUNNING COSTS UNDER DIFFERENT UNCERTAINTIES OF PV AND LOAD

\begin{tabular}{|c|c|c|c|c|}
\hline Season & No & $5 \%$ & $10 \%$ & $15 \%$ \\
\hline Summer seasons & $\$ 433.1$ & $\begin{array}{l}{[\$ 391.4,} \\
\$ 509.5]\end{array}$ & $\begin{array}{l}{[\$ 355.9,} \\
\$ 549.0]\end{array}$ & $\begin{array}{c}\$ 320.4, \\
\$ 588.6]\end{array}$ \\
\hline Winter seasons & $\begin{array}{c}\$ 493.2 \\
\$\end{array}$ & $\begin{array}{l}{[\$ 429.9} \\
\$ 604.1]\end{array}$ & $\begin{array}{l}{[\$ 394.2,} \\
\$ 644.3]\end{array}$ & $\begin{array}{l}{[\$ 358.8,} \\
\$ 684.3]\end{array}$ \\
\hline
\end{tabular}

Table III gives the simulated running costs under different load and PV uncertainties. It is easy to see that when the uncertainties increase, the running cost of $f^{+}$increases, and the running cost of $f^{-}$decreases. Fig. 5 shows the operating conditions of the PGU, exchange power with the main grid, electric chiller power, and gas boiler power during the entire scheduling horizon at different uncertainty levels in summer. 


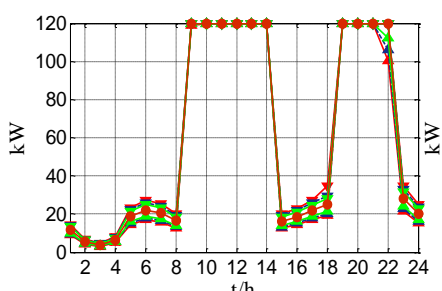

t/h

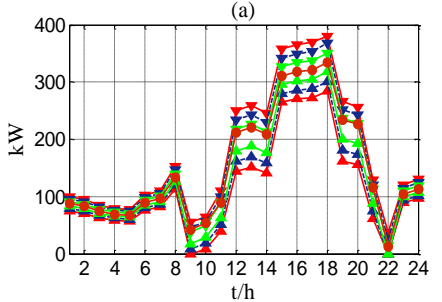

(c)

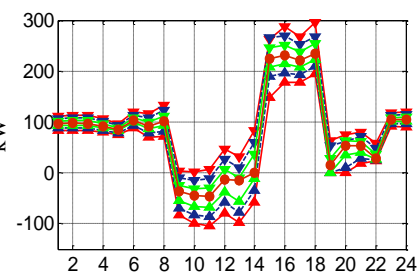

t/h

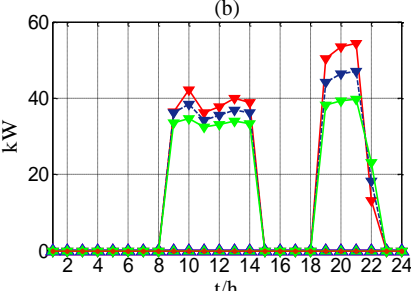

t/h $\neg$ lower bound of fluctuation $15 \% \rightarrow-$ upper bound of fluctuation $15 \%$ --1-- lower bound of fluctuation 10\%---- upper bound of fluctuation $10 \%$ $\rightarrow-$ lower bound of fluctuation $5 \% \rightarrow-$ upper bound of fluctuation $5 \%$ $\rightarrow$ No uncertainty

Fig. 5. (a) Upper and lower power of the PGU over 24 hours; (b) upper and lower exchange power with the main grid over 24 hours; (c) upper and lower power of the electric chiller over 24 hours; (d) upper and lower power of the gas boiler over 24 hours.

The exchange power with the main grid is clearly distinct, especially in Periods 9 to 14 and 19 to 20. The CCHP microgrid always sells power to the main grid in Periods 9 to 14 , and purchases power in Periods 19 to 20 when there are no load and PV fluctuations. Even under large power uncertainties, the PGU keeps its original status to guarantee normal operation. Thus, other components have to change their operating status to keep the energy balance. In Periods 9 to 14 and 19 to 21 , the CCHP microgrid may change the direction of power to cope with electricity and cooling uncertainties, and the gas boiler changes its on/off status to deal with heating uncertainties in $O C_{5 \%}^{ \pm}, O C_{10 \%}^{ \pm}$, and $O C_{15 \%}^{ \pm}$. Similar simulation results and conclusions are also obtained using a representative day in winter.

Generally, these dispatching results demonstrate that uncertainties in the heating load are mitigated by the PGU and auxiliary boilers. When the heat-electricity load ratio is low, the CCHP microgrid adjusts for uncertainties by adjusting the PGU. The CCHP microgrid also copes with gas boiler uncertainties by providing extra heat when the heat-electricity load ratio is high. Electricity load uncertainties are suppressed by the PGU and power exchange with the main grid. The power exchange depends on the uncertainty, which may even change its flow direction when the uncertainty is large. Cooling load uncertainties are restrained by the electric and absorption chillers when heating and electricity load uncertainties are suppressed. In addition, it is strongly suggested that dispatchers can give deterministic scheduling results for the PGU and battery, even when the loads and PV are highly uncertain.

\section{CONCLUSIONS}

In this paper, we developed an IMILP model for supporting the dispatching tasks of a CCHP microgrid under uncertainties. We used a CCHP microgrid in a hotel building to demonstrate the applicability of the model under three scenarios. The results indicate that IMILP can help to identify desired schemes for the dispatching results and operating conditions of equipment under uncertainties. The load and PV power uncertainties mainly affect the dispatching results of the power exchange, gas boiler and electricity chiller, while the operation of the PGU is consistent under different uncertainty levels.

In the future, because the operating condition of the PGU is consistent, it is necessary to focus on the optimization and control of other equipment in real-time scheduling. In practical applications, interval parameters for the efficiency of the PGU are relatively coarse, and the linearization of nonlinear efficiencies of PGU functions should be considered. In addition, IMILP can be further improved by considering more impact factors, and by integrating fuzzy set theory and stochastic programming methods into its framework.

\section{NOMENCLATURE}

$\pm \quad$ Lower and upper bound of interval.

$P_{\text {grid, } t}^{ \pm} \quad$ Power provided by main grid in period $t(\mathrm{~kW})$.

$P_{p g u, t}^{ \pm} \quad$ Power produced by PGU in period $t(\mathrm{~kW})$.

$P_{\text {excess }, t}^{ \pm} \quad$ Power sold to main grid in period $t(\mathrm{~kW})$.

$P_{p v, t}^{ \pm} \quad$ Power from $\mathrm{PV}$ in period $t(\mathrm{~kW})$.

$P_{e c, t}^{ \pm} \quad$ Electricity demand of electric chiller when handling the users' cooling requirement in period $t$ $(\mathrm{kW})$.

$P_{\text {disch, } t}^{ \pm} \quad$ Discharge power of battery in period $t(\mathrm{~kW})$.

$P_{c h, t}^{ \pm} \quad$ Charge of battery in period $t(\mathrm{~kW})$.

$P_{l, t}^{ \pm} \quad$ Electricity demand of users in period $t(\mathrm{~kW})$.

$W_{E S, t} \quad$ Battery energy in period $t(\mathrm{kWh})$.

$F_{m, t}^{ \pm} \quad$ Fuel consumption registered at the meter for CCHP microgrid in period $t(\mathrm{~kW})$.

$F_{p g u, t}^{ \pm} \quad$ PGU fuel consumption in period $t(\mathrm{~kW})$.

$Q_{\text {boile, }}^{ \pm} \quad$ Heat provided by the boiler in period $t(\mathrm{~kW})$.

$Q_{h r, t}^{ \pm} \quad$ Heat required by absorption chiller when handling the users' cooling requirement in period $t(\mathrm{~kW})$.

$Q_{h r h, t}^{ \pm} \quad$ Heat required by heat exchangers when handling the users' heating requirement in period $t(\mathrm{~kW})$.

$Q_{a c, t}^{ \pm} \quad$ Cooling power of the absorption chiller in period $t$ $(\mathrm{kW})$.

$Q_{e c, t}^{ \pm} \quad$ Cooling power of the electric chiller in period $t$ $(\mathrm{kW})$.

$Q_{R, t}^{ \pm} \quad$ Recovered waste heat in period $t(\mathrm{~kW})$.

$Q_{c, t}^{ \pm} \quad$ Cooling demand of the users in period $t(\mathrm{~kW})$.

$C \quad$ Unit price of electricity purchased from grid in purchase, period $t$ (\$/kWh).

$C_{\text {gas }} \quad$ Unit price of natural gas in period $t(\$ / \mathrm{kWh})$.

$C_{\text {sell,t }} \quad$ Unit price of electricity sold to grid in period $t$ (\$/kWh).

$\eta_{\text {boiler }}^{ \pm} \quad$ Auxiliary boiler efficiency.

$\eta_{p g u}^{ \pm} \quad$ PGU efficiency.

$\eta_{\text {rec }}^{ \pm} \quad$ Heat recovery system efficiency.

$\eta_{h x}^{ \pm} \quad$ Heat exchanger efficiency.

$\eta_{c h} \quad$ Charge efficiency of the battery.

$\eta_{\text {disch }} \quad$ Discharge efficiency of the battery.

$\mu \quad$ Electricity loss rate of the battery.

$C O P_{a c}^{ \pm} \quad$ Coefficient of the performance of absorption chiller.

$C O P_{e c}^{ \pm} \quad$ Coefficient of the performance of electric chiller. 


\section{REFERENCES}

[1] D. Wu and R. Wang, "Combined cooling, heating and power: A review," Prog. Energy Combust Sci., vol. 32, no. 5-6, pp. 459-495, Sep/Nov. 2006.

[2] J. Deng, R. Wang, and G. Han, "A review of thermally activated cooling technologies for combined cooling, heating and power systems," Prog. Energy Combust Sci., vol. 37, no. 2, pp. 172-203, Apr. 2011.

[3] H. Cho, P. Mago, R. Luck, and L. Chamra, "Evaluation of CCHP systems performance based on operational cost, primary energy consumption, and carbon dioxide emission by utilizing an optimal operation scheme," Applied Energy, vol. 86, no. 12, pp. 2540-2549, Dec. 2009.

[4] M. Liu, Y. Shi, and F. Fang, "A new operation strategy for CCHP systems with hybrid chillers," Applied Energy, vol. 95, no. 7, pp. 164-173, Jul. 2012

[5] X. Zhang, G. G. Karady, and S. T. Ariaratnam, "Optimal allocation of CHP-based distributed generation on urban energy distribution networks," IEEE Trans. Sustainable Energy, vol. 5, no. 1, pp. 246-253, Jan. 2014.

[6] P. Arcuri, G. Florio, and P. Fragiacomo, "A mixed integer programming model for optimal design of trigeneration in a hospital complex," Energy, vol. 32, no. 8, pp. 1430-1447, Aug. 2007.

[7] W. Gu, Z. Wu, R. Bo, W. Liu, G. Zhou, and W. Chen, "Modeling, planning and optimal energy management of combined cooling, heating and power microgrid: A review," International Journal of Electrical Power \& Energy Systems, vol. 54, no. 8, pp. 26-37, Jan. 2014.

[8] Z. Wu, W. Gu, R. Wang, X. Yuan, and W. Liu, "Economic optimal schedule of CHP microgrid system using chance constrained programming and particle swarm optimization," Power and Energy Society General Meeting, vol. 35, no. 8, pp. 1-11, Jul. 2011.

[9] F. Salgado and P. J. Pedrero, "Short-term operation planning on cogeneration systems: A survey," Electric Power Systems Research, vol. 78, no. 5, pp. 835-848, May 2012.

[10] S. Sanaye and A. Nasab, "Modeling and optimizing a CHP system for natural gas pressure reduction plant," Energy, vol. 40, no. 1, pp. 358-369, Apr. 2012.

[11] H. Cho, R. Luck, S. Eksioglu, and L. Chamra, "Cost-optimized real-time operation of CHP systems," Energy and Buildings, vol. 41, no. 4, pp. 445-451, Apr. 2009.

[12] G. S. Piperagkas, A. G. Anastasiadis, and N. D. Hatziargyriou, "Stochastic PSO-based heat and power dispatch under environmental constraints incorporating CHP and wind power units," Electric Power Systems Research, vol. 81, no. 1, pp. 209-218, Jan. 2011.

[13] P. Mancarella and G. Chicco, "Assessment of the greenhouse gas emissions from cogeneration and trigeneration systems. Part II: analysis techniques and application cases," Energy, vol. 33, no. 3, pp. 418-430, Mar. 2008.

[14] J. Liu, Q. Lin, G. H. Huang, Q. Wu, and H. P. Li, "Energy systems planning and GHG-emission control under uncertainty in the province of Liaoning, China-A dynamic inexact energy systems optimization model," International Journal of Electrical Power \& Energy Systems, vol. 53, no. 12, pp. 142-158, Dec. 2013.

[15] X. Liu, "Optimization of a combined heat and power system with wind turbines," International Journal of Electrical Power \& Energy Systems, vol. 43, no. 1, pp. 1421-1426, Dec. 2012.

[16] L. Wang and C. Singh, "Stochastic combined heat and power dispatch based on multi-objective particle swarm optimization," International Journal of Electrical Power \& Energy Systems, vol. 30, no. 3, pp. 226-234, Mar. 2008.

[17] A. C. Marquez A. S. Heguedas, and B. Lung, "Monte Carlo-based assessment of system availability. A case study for cogeneration plants," Reliability Engineering \& System Safety, vol. 88, no. 3, pp. 273-289, Jun. 2005.

[18] J. Wu, J. Zhu, G. Chen, and H. Zhang, "A hybrid method for optimal scheduling of short-term electric power generation of cascaded hydroelectric plants based on particle swarm optimization and chance-constrained programming," IEEE Trans. Power Sys., vol. 23, no. 4 , pp. $1570-1579$, Nov. 2008.

[19] Z. Liu, F. Wen, and G. Ledwich, "Optimal siting and sizing of distributed generators in distribution systems considering uncertainties," IEEE Trans. Power Delivery, vol. 26, no. 4, pp. 2541-2551, Oct. 2011

[20] M. Moradi, M. Hajinazari, S. Jamasb, and M. Paripour, "An energy management system (EMS) strategy for combined heat and power
(CHP) systems based on a hybrid optimization method employing fuzzy programming," Energy, vol. 49, pp. 86-101, Jan. 2013.

[21] A. Smith, R. Luck, and P. J. Mago, "Analysis of a combined cooling, heating, and power system model under different operating strategies with input and model data uncertainty," Energy and Buildings, vol. 42 , no. 11, pp. 2231-2240, Nov. 2010.

[22] T. Rezvan, N. S. Gharneh, and G. B. Gharehpetian, "Optimization of distributed generation capacities in buildings under uncertainty in load demand," Energy and Buildings, vol. 57, no. 1, pp. 58-64, Feb. 2013.

[23] X. S. Qin, G. H. Huang, G. M. Zeng, A. Chakma, and Y. F. Huang, "An interval-parameter fuzzy nonlinear optimization model for stream water quality management under uncertainty," European Journal of Operational Research, vo1. 80, no. 3, pp. 1331-1357, Aug. 2007.

[24] Y. F. Li, Y. P. Li, G. H. Huang, and X. Chen, "Energy and environmental systems planning under uncertainty - An inexact fuzzy-stochastic programming approach," Applied Energy, vol. 87, no 10, pp. 3189-3211, Oct. 2010.

[25] G. C. Li, G. H. Huang, Q. G. Lin, X. D. Zhang, Q. Tan, and Y. M. Chen. "Development of a GHG-mitigation oriented inexact dynamic model for regional energy system management," Energy, vol. 36, no. 5, pp. 3388-3398, May 2011

[26] Y. P. Li, G. H. Huang, and X. Chen, "Planning regional energy system in association with greenhouse gas mitigation under uncertainty," Applied Energy, vol. 88, no. 3, pp. 599-611, Mar. 2011.

[27] T. Ding, R. Bo, W. Gu, Q. Guo, and H. Sun, "Absolute value Constraint based method for interval optimization to SCED model," IEEE Trans. Power Sys.

[28] Y. R. Fan and G. H. Huang, "A robust two-step method for solving interval linear programming problems within an environmental management context," J. Environ Inform, vol. 19, no. 1, pp. 1-12, Mar. 2012.

[29] G. H. Huang, B. W. Baetz, and G. Patry, "Grey integer programming: an application to waste management planning under uncertainty," European Journal of Operational Research, vol. 83, no. 3, pp 594-620, Jun. 1995.

[30] G. Li, G. H. Huang, Q. Lin, Y. Cai, Y. Chen, and X. Zhang, "Development of an interval multi- stage stochastic programming model for regional energy systems planning and GHG emission control under uncertainty," International Journal of Energy Research, vol. 36 , no. 12, pp. 1161-1174, Oct. 2012.

[31] U. S Department of Energy, Energy Plus. [Online]. Available: http://www.energyplus.gov

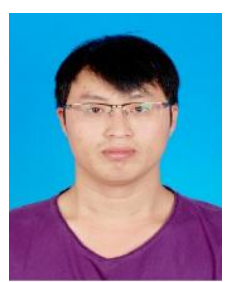

Zhao Luo received his M.Eng degree in electrical engineering from Southeast University, China, in 2013. He is currently pursuing a Ph.D. degree in Electrical engineering at Southeast University. His research interests include renewable energy technology and micro-grids.

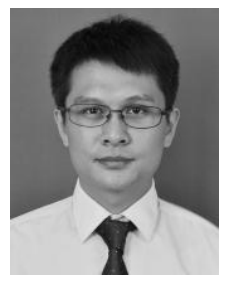

Wei Gu received his B.Eng. and Ph.D. degrees in electrical engineering from Southeast University, China, in 2001 and 2006, respectively.

From 2009 to 2010, he was a visiting scholar in the Department of Electrical Engineering, Arizona State University, Tempe, AZ, USA. He is now a professor in the School of Electrical Engineering at Southeast University. His research interests include distributed generation and micro-grids, active distribution networks, and power quality.

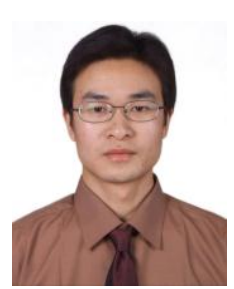

Song Gao received an M.Eng degree in electrical engineering from North China Electric Power University. He is now an engineer in Jiangsu Electric Power Company. His research interests are renewable energy technology and distribution network planning. 


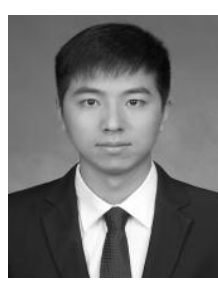

Zhihe Wang received a B.Eng. degree in electrical engineering from China University of Mining and Technology, China, in 2013. He is currently pursuing an M.Eng degree in electrical engineering at Southeast University, China. His research interests are renewable energy technology and economic dispatch of micro-grids.

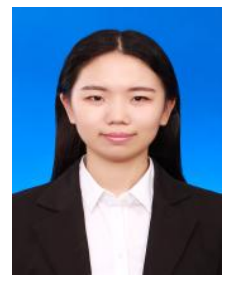

Yiyuan Tang received a B.Eng. degree in electrical engineering from Southeast University, China, in 2013. She is currently pursuing an M.Eng degree in electrical engineering at Southeast University, China. Her research interests are renewable energy technology and economic dispatch of micro-grids. 